\title{
Estudio del consumo de azúcares reductores durante la fermentación alcohólica del mosto de uva italia para la obtención de vino blanco
}

ReCibidO: 13/06/2016 AcEPTADO: 16/11/2016

\author{
Roberto Robles Calderón* \\ OSIRIS FeliCIANo MuÑoZ** \\ Jaqueline Heidy Chirre Flores $* * *$
}

\begin{abstract}
RESUMEN
En la elaboración de vino blanco es importante controlar el tiempo de la fermentación alcohólica del mosto, debido a que si el tiempo es menor, la fermentación es incompleta, obteniéndose un vino con baja graduación alcohólica a causa de que no se consume la totalidad de los azúcares reductores presentes en el mosto y además no se desarrollan adecuadamente los delicados aromas y sabores afrutado característicos de un vino blanco.

La presente investigación tiene por objetivo determinar el tiempo de fermentación anaeróbica, mediante el consumo de azucares reductores, inoculando el mosto con $4 \mathrm{~g} / \mathrm{L}$ de cepas de levadura Saccharomyces Cerevisiae (Lalvin D 47), manteniendo la fermentación a las siguientes condiciones: acidez $6,5 \mathrm{~g} / \mathrm{L}$, temperatura a $16{ }^{\circ} \mathrm{C}$ y fosfato de amonio $0,2 \mathrm{~g} / \mathrm{L}$ (nutriente). Se utilizó como materia prima uva italia, proveniente de la provincia de Cañete. Las condiciones iníciales del mosto han sido: grados Brix, 22; acidez, 5,7 g/L. El proceso de fermentación se realizó en un envase de vidrio de $10 \mathrm{~L}$, al cual se adaptó una trampa de gas para la salida del $\mathrm{CO}_{2}$ que se forma durante la fermentación. Para mantener la temperatura de fermentación, se utilizó un equipo de frío. El tiempo de fermentación a las condiciones indicadas anteriormente ha sido de 21 días, obteniéndose un vino con azúcar residual de $1 \%$, aromático y de buena calidad.

Palabras clave: fermentación anaeróbica, grados Brix, Saccharomyces Cerevisiae
\end{abstract}

STUDY OF THE CONSUMPTION OF REDUCING SUGARS DURING THE ALCOHOLIC FERMENTATION OF THE ITALIAN GRAPE MUST TO OBTAIN WHITE WINE

\section{ABSTRACT}

This research aims to determine the time of anaerobic fermentation by reducing sugar intake, inoculating the wort with $4 \mathrm{~g} / \mathrm{L}$ of Saccharomyces Cerevisiae yeast strains (Lalvin D 47), keeping the fermentation to the following conditions: Acidity $6.5 \mathrm{~g} / \mathrm{L}$, temperature $16^{\circ} \mathrm{C}$ and Ammonium phosphate $0,2 \mathrm{~g} / \mathrm{L}$ (nutrient). It was used as raw material grape Italy, from the Province of Cañete. The initial conditions must have been: Brix, 22; acid, $5.7 \mathrm{~g} / \mathrm{L}$.

The fermentation process was performed in a glass container of $10 \mathrm{~L}$, to which a gas trap adapted to output the $\mathrm{CO} 2$ formed during fermentation. To maintain the fermentation temperature, cooling equipment was used. The fermentation time to the above conditions has been 21 days, yielding a wine with residual sugar $1 \%$, aromatic and of good quality.

Keywords: anaerobic fermentation, brix degree, Saccharomyces Cerevisiae

\section{INTRODUCCIÓN}

El vino es el más complejo de los productos agrícolas. Ningún otro es capaz de expresar tantos matices sensorialmente palpables. Estos son consecuencia de muchos factores, fundamentalmente del tipo de suelo, las condiciones climatológicas, la variedad de uva o viña empleadas y las prácticas vinícolas aplicadas, (Bison, 2001).

Los vinos blancos son más delicados, y las condiciones de elaboración son diferentes a la de vinos tintos; siendo las condiciones de fermentación alcohólica las siguientes: temperatura $<20^{\circ} \mathrm{C}$ y acidez entre $6,0 \mathrm{~g} / \mathrm{L}$ y $6,5 \mathrm{~g} / \mathrm{L}$ (Butzke, 2010).

La presente investigación tiene por objetivo determinar el tiempo de fermentación mediante el consumo de azucares reductores, manteniendo la acidez entre $6,0 \mathrm{~g} / \mathrm{L}$ y $6,5 \mathrm{~g} / \mathrm{L}$ (como ácido tartárico), la temperatura de fermentación de $16{ }^{\circ} \mathrm{C}$ y a la concentración de nutriente (fosfato de amonio) de 0,2 $\mathrm{g} / \mathrm{L}$ (Butzke, 2010).

\section{DESCRIPCIÓN DE LA MATERIA PRIMA}

\section{A. Clima y suelos}

El suelo es uno de los factores más críticos que afectan el rendimiento de la vid. El suelo tiene muchos efectos. No solo proporciona nutrientes para las plantas, sino que también determina la cantidad de agua retenida en ese suelo, y la cantidad de agua que por lo tanto está disponible para las raíces. La composición del suelo también influirá en los microorganismos, principalmente bacterias y hongos, capaces de interactuar con la estructura de la raíz de la planta. Algunos de estos organismos son beneficiosos para la planta, algunos son neutrales y algunos son perjudiciales. La composición del suelo no solamente afecta la naturaleza de los organismos presentes, también afecta sus interacciones (Bison, 2001).

Las condiciones climáticas, particularmente la temperatura, de la región donde han crecido las uvas tienen efecto decisivo en

\footnotetext{
* Ing. Químico, Docente FQIQ. UNMSM. E-mail: robertoc-r@hotmail.com

** Ing. Industrial, Docente FII. UNMSM. E-mail:Osiris.fm@hotmail.com

*** Ing. Petroquímico, Empresa HIDROLED. E-mail: jaqui chirreflores@yahoo.es
} 
la relación de azúcar a acidez, el incremento de la temperatura causa un correspondiente incremento de esta relación. Se sabe que la acidez disminuye mientras que la uva se vuelve más madura. El estado de madurez al cual las uvas fueron cosechadas, tiene una decidida influencia en la conveniencia de la uva para producir un tipo de vino dado (Bison, 2001).

\section{B. Variedades de uvas en el Perú}

\section{Uvas para mesa}

Red Globe, Negra Corriente, Alfonso Lavallete, Italia, Quebranta, Borgoña, etc.

\section{Uvas para vinos y pisco}

- Para vinos tintos y rosados: Quebranta, Malbeck, Cabernet, Borgoña negra.

- Para vinos blancos: Italia, Borgoña blanca, Pinot, Albilla, Torontel.

- Para Pisco: Quebranta, Italia, Moscatel, Negra corriente, albilla, Uvina.

- Variedades aromáticas: Italia, Albilla, Torontel, Moscatel.

- Variedades no aromáticas: Quebranta, Negra Criolla, Uvina, Mollar.
En la Tabla 1, se describe los aromas característicos y el país de origen de algunas variedades de uva blanca.

\section{ACIDEZ DE LA UVA Y DEL VINO}

La acidez en el vino se debe a varios ácidos orgánicos derivados de la uva y los producidos durante la fermentación. Los ácidos Tartárico, málico y cítrico se producen en las uvas y los ácidos succínico, láctico y acético se forman durante la fermentación. Durante la maduración el contenido de ácido de las uvas disminuyen y el $\mathrm{pH}$ sube. La reducción de la acidez y posterior incremento del $\mathrm{pH}$ se debe principalmente a la degradación del ácido málico (especialmente mayor en climas calientes) y la neutralización de ácidos por los cationes adicionados, sobre todo de potasio. La decisión de la cosecha de la fruta se hace cuando la acidez, $\mathrm{pH}$ y la concentración de azúcar alcanzan un nivel deseado.

El pH del mosto y/o vino generalmente oscila entre 3,0 y 4,0 , pero los valores de $\mathrm{pH}$ en el rango de 3.2 a 3.6 son más comunes (Stiener, 2001).

En la Tabla 2, se indica los principales ácidos de la uva y vino con su estructura molecular, peso molecular, peso equivalente y valores de pKa.

Tabla 1. Términos descriptivos del aroma para diferentes variedades de uva blanca

\begin{tabular}{|c|c|c|}
\hline Variedad de uva & País de origen & Descripción de aromas \\
\hline Chardonay & Fancia & Manzana, melón, durazno, almendra \\
\hline Chenin Blanc & Francia & Camelia, guayaba \\
\hline Garganega & España & Citronela, condimento \\
\hline Gewürztraminer & Italia & Moscatel. \\
\hline Muscat & Grecia & Cítrico, manzana verde, licor \\
\hline Parellada & España & Fruta, queso romano \\
\hline Pinot Gris & Francia & Rosa, pino, fruta \\
\hline Riesling & Alemania & Durazno \\
\hline Rousanne & Francia & Pimiento, floral, herbáceo \\
\hline Sauvignon Blanc & Francia & Higo, melón \\
\hline Semillon & Francia & Manzana verde \\
\hline Torbato & Italia & Durazno, albaricoque \\
\hline Viognier & España & Vainilla, plátano \\
\hline Viura & Francia &
\end{tabular}

Fuente: Calo`et al. (2008). 
Tabla 2. Principales ácidos de la uva y vino

\begin{tabular}{|l|l|l|l|l|l|}
\hline \multicolumn{1}{|c|}{ Acido } & \multicolumn{1}{c|}{ Estructura molecular } & $\begin{array}{c}\text { Peso } \\
\text { molecular }\end{array}$ & $\begin{array}{c}\text { Peso } \\
\text { equivalente }\end{array}$ & \multicolumn{1}{c|}{ Pka1 } & \multicolumn{1}{c|}{ Pka2 } \\
\hline Tartárico & $\mathrm{COOH}-(\mathrm{CHOH}) 2-\mathrm{COOH}$ & 150,1 & 75 & 3,04 & 4,34 \\
\hline Málico & $\mathrm{COOH}-\mathrm{CH} 2-\mathrm{CHOH}-\mathrm{COOH}$ & 134,1 & 67 & 3,46 & 5,1 \\
\hline Cítrico & $\mathrm{CH} 2-\mathrm{COOH}-\mathrm{CHOCOOH}-\mathrm{CH} 2 \mathrm{COOH}$ & 192,1 & 64 & 3,13 & 4,74 \\
\hline Láctico & $\mathrm{COOH}-\mathrm{CHOH}-\mathrm{CH} 3$ & 90,1 & 90 & 3,86 & - \\
\hline Succínico & $\mathrm{CH} 2-\mathrm{COOH}-\mathrm{CH} 2-\mathrm{COOH}$ & 118,1 & 59 & 4,21 & 5,64 \\
\hline Acético & $\mathrm{CH} 3-\mathrm{COOH}$ & 60,1 & 60 & 4,75 & - \\
\hline
\end{tabular}

Valores de pka a $25^{\circ} \mathrm{C}$

Fuente: Adaptado de Margalit (1997).

\section{TEMPERATURA DE FERMENTACIÓN}

Las altas temperaturas de fermentación estimulan rápidas tasas de metabolismo acelerando la producción de etanol, y conducen a una mayor pérdida de caracteres volátiles.

Sin embargo, si la fermentación se vuelve demasiado caliente, el crecimiento y metabolismo de la Saccharomyces se inhibirán. Por lo tanto, el impacto de una mala elección de la temperatura durante la fase más activa de la fermentación, puede afectar la concentración de etanol al final de la fermentación y la capacidad de la levadura para consumir todo el azúcar disponible (Bison, 2001).

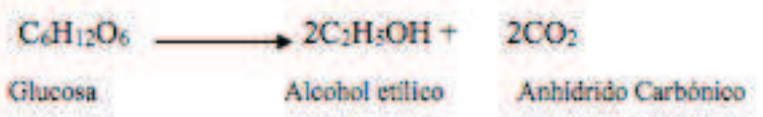

\section{PROCESO}

A. Determinación del peso promedio de los racimos:

La Figura 1 muestra 3 racimos de uva italia tomados a azar, cantidad de escobajo y la Tabla 3 indica el peso promedio de los 3 racimos.

- Rendimiento Promedio de los 3 racimos de uva Italia: La Figura 2 muestra las uvas buenas y en mal estado y la obtención del jugo, respectivamente.

Figura 1. A, Tres racimos diferentes de uva Italia; B, rendimiento de escobajo.

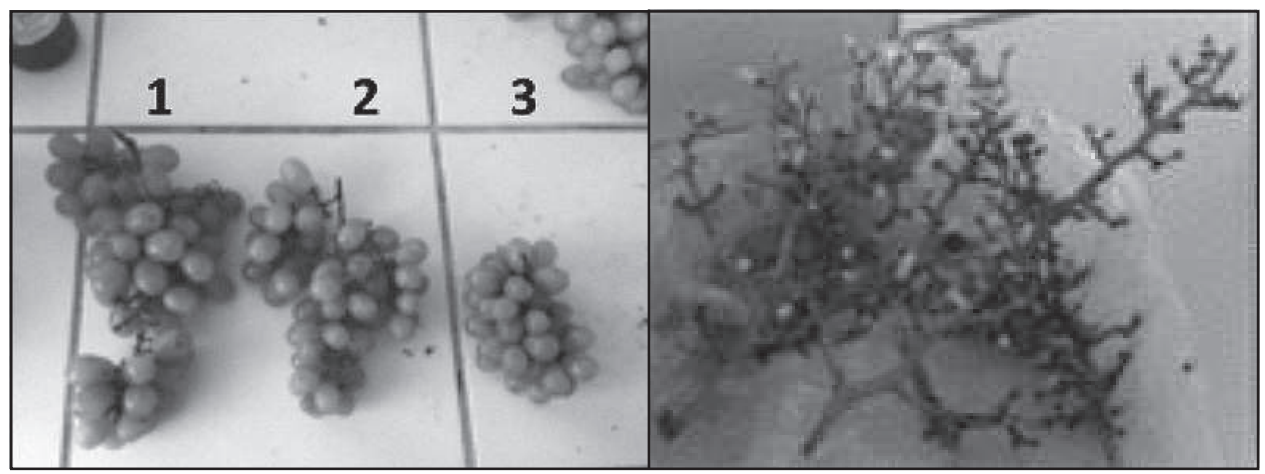

A

B

Fuente: Elaboración propia. 
Tabla 3. Peso promedio de 3 racimos de uva italia

\begin{tabular}{|c|c|c|}
\hline Racimo & Peso $(\mathbf{k g})$ & Peso promedio $(\mathbf{k g})$ \\
\hline 1 & 0.556 & \\
\hline 2 & 0.368 & \multirow{2}{*}{0.406} \\
\hline 3 & 0.296 & \\
\hline
\end{tabular}

Fuente: Elaboración propia.

Figura 2. A, uvas en buen estado; B, uvas en mal estado; C, obtención del jugo.

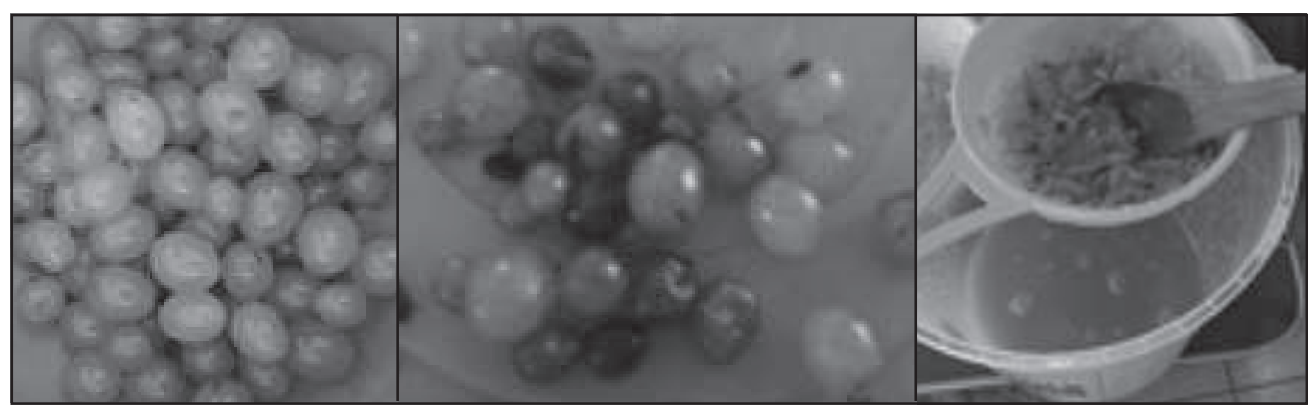

A

B

C

Fuente: Elaboración propia.

Tabla 4. Rendimiento y grados Brix promedio de 3 racimos de uva italia

\begin{tabular}{|c|c|c|c|c|c|}
\hline Características & Jugo de uva & Cáscara y pepas & Pérdidas & Escobajo & Rendimiento \% \\
\hline Peso (kg) & 0.291 & 0.09 & 0,01 & 0,015 & \multirow{4}{*}{71,6} \\
\hline Porcentaje & 71,6 & 22,2 & 2,5 & 3,7 & \\
\hline${ }^{\circ}$ Brix & \multicolumn{2}{|c|}{21} & & & \\
\hline Total & \multicolumn{2}{|c|}{0.406} & & & \\
\hline
\end{tabular}

Fuente: Elaboración propia.

En la Tabla 4, se indica el rendimiento promedio de 3 racimos de uva Italia.

\section{B. Descripcion del proceso}

La Tabla 5, indica el rendimiento promedio de 3 lotes de $10 \mathrm{~kg}$ de uva Italia, como se puede apreciar el rendimiento de jugo es alto.

\section{Materia prima}

Uva italia: $10 \mathrm{~kg}$

\section{Selección}

En esta etapa se separa las uvas verdes, y las que se encuentran en mal estado.
Materia Prima: $10 \mathrm{~kg}$

Pérdidas $160 \mathrm{~g}(1.6 \%)$

\section{Estrujado}

Se separa el escobajo del jugo y cáscara.

Materia prima: $9,84 \mathrm{~kg}$

Pérdida (escobajo): $470 \mathrm{~g}(4,7 \%)$

\section{Fermentación}

Es la etapa más importante en el proceso de vino o Pisco.

Las cáscaras de uva italia contienen azúcares, aromas y sabores, por lo que es necesario realizar 
la fermentación del jugo junto éstas, para extraer dichos componentes, durante las primeras $24 \mathrm{~h}$.

Materia prima (Jugo + cáscara + Semillas): 9,37 kg

- Activación de la levadura: se hierve $100 \mathrm{~mL}$ agua en un vaso Pírex de $250 \mathrm{~mL}$, se enfría a $32{ }^{\circ} \mathrm{C}$, se agrega la levadura y se deja que se active durante 10 minutos (Figura 3 )

- Nutriente: fosfato di amónico, a la concentración de $0,2 \mathrm{~g} / \mathrm{L}$.

- Metabisulfito de potasio: se agrega a la concentración de 75 ppm, para eliminar microorganismos que puedan interferir la acción de las levaduras.

- Se corrige la acidez si no está en el rango adecuado.

- Luego de realizar las correcciones, se agrega la levadura, se coloca la trampa de gas al fermentador y se fermenta a $16{ }^{\circ} \mathrm{C}$.

\section{Primer trasvase}

El vino blanco, tiene aromas y sabores delicados, por lo que es necesario retirar las cáscaras y semillas del mosto después de $24 \mathrm{~h}$ de fermentación, para evitar que extraiga los taninos de las cáscaras y semillas.

Mosto (cáscara + semillas): 9,37 kg

Pérdidas, (cáscaras + semillas): 2,34 kg

\section{Fermentación}

Mosto: 7,03 kg

En esta etapa, se realiza el control de acidez para una buena fermentación, agregando ácido tartárico o carbonato de calcio para mantener la acidez dentro del rango adecuado de $6,0 \mathrm{~g} / \mathrm{L}$ a 6,5 $\mathrm{g} / \mathrm{L}$ (como ácido tartárico). También se controla la temperatura, el cual debe estar alrededor de $16{ }^{\circ} \mathrm{C}$.

A continuación, la Figura 4 resume el proceso de fermentación alcohólica.

Tabla 5. Rendimiento de uva italia

\begin{tabular}{|c|l|l|l|l|l|}
\hline Muestra & Peso $(\mathbf{k g})$ & \multicolumn{1}{|c|}{ Pérdidas $(\mathbf{k g})$} & \multicolumn{1}{|c|}{ Escobajo (kg) } & $\begin{array}{c}\text { Cáscara y } \\
\text { semillas }(\mathbf{k g})\end{array}$ & Jugo (kg) \\
\hline 1 & 10 & 0,2 & 0,42 & 2,6 & 6,8 \\
\hline 2 & 10 & 0,13 & 0,51 & 2,2 & 7,2 \\
\hline 3 & 10 & 0,15 & 0,48 & 2,1 & 7,3 \\
\hline PROMEDIO & 10 & 0,16 & 0,47 & 2,3 & 7,1 \\
\hline Porcentaje & 100 & 1.6 & 4,7 & 25 & 71 \\
\hline
\end{tabular}

Fuente: Elaboración propia.

Figura 3. A, Levadura en polvo; B, Levadura activada; C, Mosto.

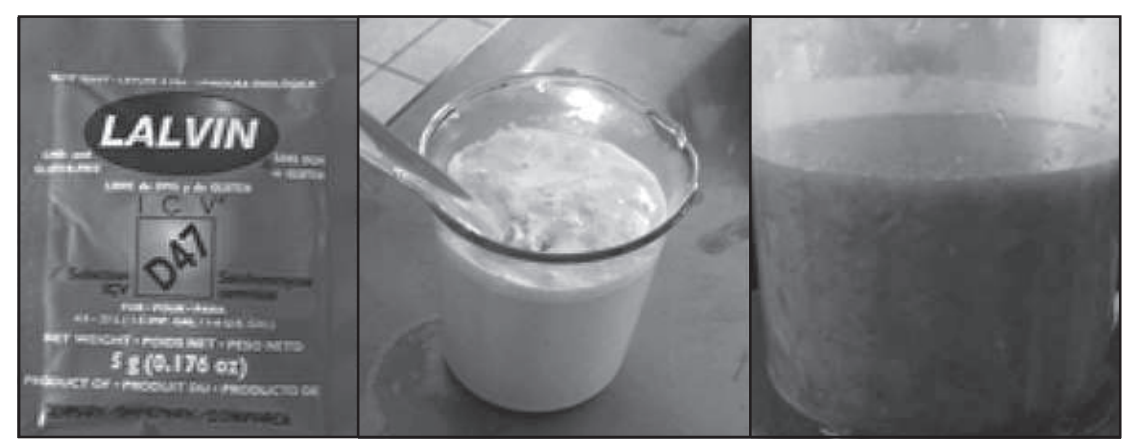

A

B

C

Fuente: Elaboración propia. 
Figura 4. Diagrama de flujo del proceso de fermentación alcohólica.

UVA

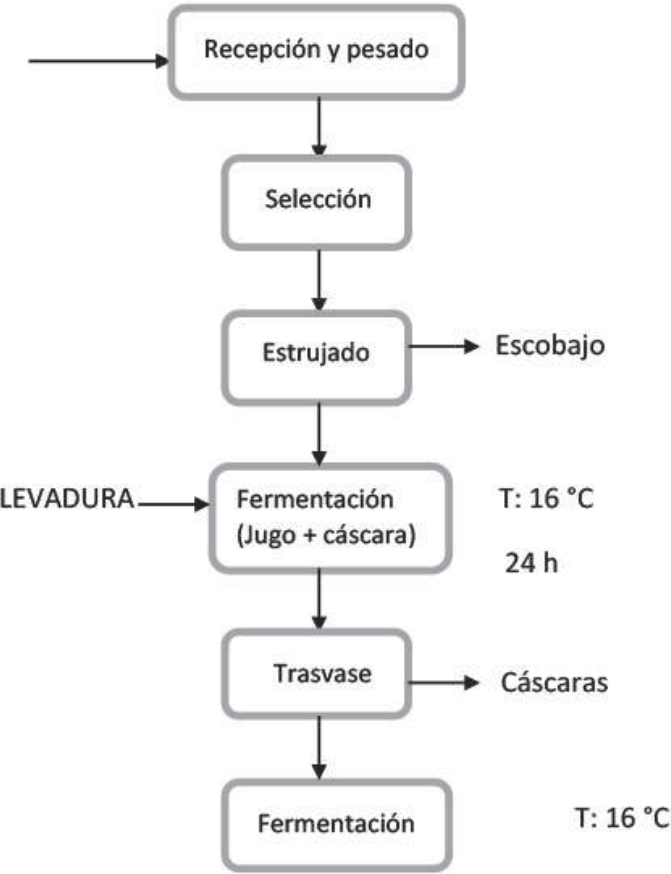

Fuente: Elaboración propia
La Tabla 6 indica la variación en porcentajes de la acidez y azucares reductores durante la fermentación alcohólica, también se aprecia que la concentración de azúcares reductores, disminuye rápidamente los primeros días de fermentación.

La Figura 5 muestra la variación de la concentración de azúcares reductores (para la muestra 3), en el mosto con el tiempo de fementación alcohólica, se puede apreciar que durante los primeros 5 días el consumo de azúcares es mayor, debido a que los primeros días la velocidad de reacción es más alta. También nos indica que a los 21 días, se ha consumido casi todo el azúcar fermentable, lo que indica que el proceso de fermentación ha terminado.

Figura 5. Consumo de azúcares reductores durante la fermentación alcohólica.

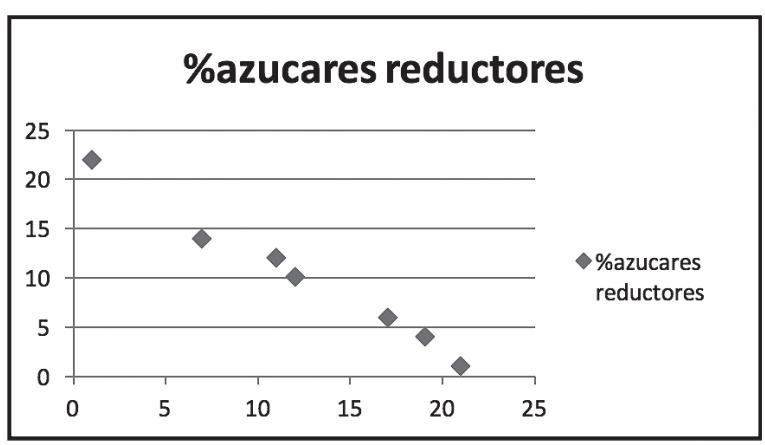

Fuente: Elaboración propia.

Tabla 6. Consumo de azúcares reductores y acidez durante el proceso de fermentación alcohólica del mosto de uva italia

\begin{tabular}{|c|c|c|c|c|c|c|}
\hline \multirow{2}{*}{ Tiempo (días) } & \multicolumn{3}{|c|}{ \% Acidez } & \multicolumn{3}{c|}{ \% Azúcares reductores } \\
\cline { 2 - 7 } & 1 & 2 & 3 & 1 & 2 & 3 \\
\hline 1 & 0,75 & 0,67 & 0,68 & 23 & 20 & 22 \\
\hline 7 & 0,58 & 0,59 & 0,54 & 18,1 & 14,6 & 14 \\
\hline 11 & 0,72 & 0.66 & 0,65 & 14,8 & 10.7 & 12 \\
\hline 12 & 0,71 & 0,69 & 0,68 & 8,4 & 7,1 & 10 \\
\hline 17 & 0,74 & 0,64 & 0.70 & 7,8 & 6,5 & 6 \\
\hline 19 & 0,78 & 0.60 & 0.67 & 6,1 & 4,4 & 4 \\
\hline 21 & 0,67 & 0.63 & 0.62 & 4,1 & 3,4 & 1 \\
\hline
\end{tabular}

Fuente: Elaboración propia. 
La Figura 6 muestra que la concentración de acidez del mosto (para la muestra 3), durante la fermentación se mantiene dentro del rango adecuado $(0,6 \%$ a $0,7 \%)$; Esto se logra agregando ácido tartárico o carbonato de calcio, según que la acidez disminuya o aumente, respectivamente.

Figura 6. Control de acidez durante el proceso de fermentación alcohólica.

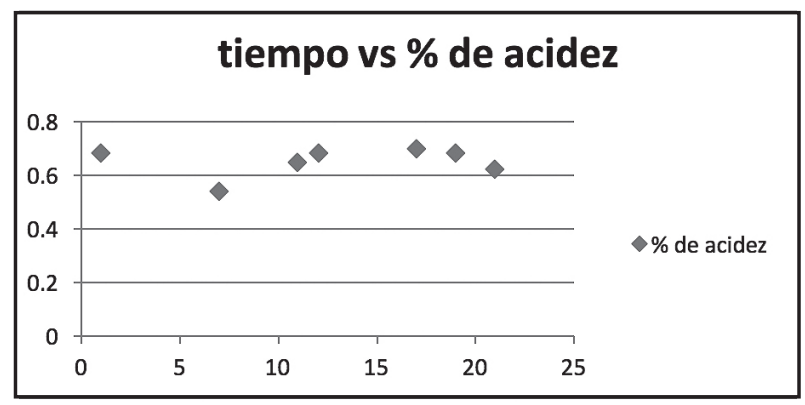

Fuente: Elaboración propia.

\section{CONCLUSIONES}

1. Debido a que la fermentación de mostos de uvas blancas se lleva a cabo a $16{ }^{\circ} \mathrm{C}$, el tiempo de fermentación alcohólica es de 21 días, esto se debe a que la velocidad de reacción es menor a bajas temperaturas.
2. En la Figura 6 se puede observar que la acidez del mosto cambia durante el proceso de fermentación alcohólica principalmente debido a las reacciones que suceden y a la formación de ácido láctico, succínico y acético, por lo que es necesario mantener la concentración del mosto entre $6,0 \mathrm{~g} / \mathrm{L}$ y $6,5 \mathrm{~g} / \mathrm{L}$, agregando carbonato de calcio o ácido tartárico según que la variación de acidez del mosto sea alta o baja, respectivamente.

\section{REFERENCIAS BIBLIOGRÁFICAS}

[1] Calo' A, (2008). Costacurta, A., Maraš, V., Meneghetti, S., and Crespan, M. (2008). Molecular correlation of Zinfandel (Primitivo) with Austrian, Croatian, and Hungarian cultivars and Kratos ija, an additional synonym. Am. J. Enol. Vitic. 59, 205-209.

[2] Butzke C., (2010). Winemaking Problems Solved, New York, USA: Woodhead Publishing limited.

[3] Margalit, Y. (1997). Concepts in wine chemistry. San Francisco, USA: Wine Appreciation Guild

[4] Stiener T., (2001). Ohio Grape - Wine, sponsoredbyhorticulture and cropscience, Ohio, USA: The Ohio StateUniversity.

[5] Bison L., (2001). Factors Influencing Wine Quality. University of California at Davis, University Extensión. 